\title{
Acute Pancreatitis Related to a Chemotherapy Drug
}

\author{
Ghulam Murtaza ${ }^{\mathrm{a}, \mathrm{c}}$, Anadil Faqah ${ }^{\mathrm{a}}$, Nicholas Konowitz ${ }^{\mathrm{b}}$, Hannah Lu ${ }^{\mathrm{b}}$, \\ Aneesh Kuruvilla ${ }^{a}$, Sujeen Adhikari ${ }^{\mathrm{a}}$
}

\begin{abstract}
Drug-induced acute pancreatitis is a rare cause of pancreatitis. We present a case of pancreatitis caused by pazopanib, a tyrosine kinase inhibitor used in the treatment of renal cell carcinoma. A 57-year-old male with no risk factors for pancreatitis and a past medical history of renal cell carcinoma who was being treated with pazopanib presented with epigastric pain with radiation to the back. Lipase was elevated to 7,960 units/L. Pazopanib was discontinued on arrival and his lipase levels decreased from 7,960 to 3,380 units/L one day after discontinuation. Abdominal pain resolved and patient tolerated a diet. This case illustrates the importance that medical professionals should be aware of acute pancreatitis as a rare but severe side effect of pazopanib and therefore should monitor and educate their patients accordingly.
\end{abstract}

Keywords: Abdominal pain; Pancreatitis; Renal cell carcinoma; Pazopanib

\section{Introduction}

Acute pancreatitis is a common diagnosis in the inpatient setting and it is estimated that 300,000 patients per year are affected [1]. The diagnosis is made by the presence of two of the three following criteria: abdominal pain consistent with disease, serum amylase or lipase levels greater than three times the upper limit of normal, or characteristic findings on abdominal imaging. Gallstones and alcohol are the most common causes of pancreatitis and account for $75 \%$ of the cases but medications can also cause pancreatitis $[1,2]$. Acute pancreatitis induced by pazopanib, an oral multitargeted tyrosine kinase inhibitor (TKI) used in the treatment of renal cell carcinoma, has been rarely reported as a side effect [3]. We present an interesting case of pazopanib-induced pancreatitis.

Manuscript accepted for publication February 07, 2017

aDepartment of Internal Medicine, Advocate Christ Medical Center, Oak Lawn, IL, USA

${ }^{b}$ Rosalind Franklin University of Medicine and Science, North Chicago, IL, USA

${ }^{\mathrm{c} C}$ Corresponding Author: Ghulam Murtaza, Department of Internal Medicine, Advocate Christ Medical Center, 4440 W 95th St, Oak Lawn, IL, USA.

Email: ghlmurtaza@gmail.com

doi: https://doi.org/10.14740/wjon1006e

\section{Case Report}

A 57-year-old male with past medical history of metastatic left renal cell carcinoma and a right kidney mass confirmed by biopsy who was started on pazopanib 6 months ago presented with abdominal pain. The epigastric pain started one night prior and improved with Tylenol. The pain was 9/10 in severity, not associated with eating and radiated to his back. Patient denied having an appetite and endorsed a few episodes of non-bilious vomiting as well as nausea. The patient was recently seen by the oncologist in the outpatient setting because he was having side effects from pazopanib including fatigue and few episodes of watery diarrhea per day. As a result, the patient was only taking two pills of pazopanib $100 \mathrm{mg}$ daily instead of the prescribed 400 mg twice daily. Outpatient CT scan of the abdomen did show improvement and stability of the disease. Hence, he was asked to start taking three pills instead of two and to slowly uptitrate.

On presentation in the emergency department, liver function tests were normal: alanine transaminase 10 units/L, aspartate transaminase 18 units/L, alkaline phosphatase 63 unit/L. Calcium level was normal. Triglycerides were not elevated and cholesterol was normal. Lipase was elevated to 7,960 units/L (reference range 73 - 393 unit/L). Basal metabolic panel was also normal. Patient did not drink alcohol and did not have any risk factors for pancreatitis. Pazopanib was discontinued the same day the patient presented with abdominal pain and his lipase level decreased from 7,960 to 3,380 units/L one day after discontinuation. He was able to tolerate a clear liquid diet which was progressed to a low residual diet. He was sent on home tramadol and was asked to follow-up with oncology to determine a new treatment regimen for his renal cell carcinoma.

\section{Discussion}

Well-known adverse effects of TKIs are hyperamylasemia and hyperlipidemia [3]. The patient in our case presented with acute onset abdominal pain radiating to the back with a lipase level much greater than three times the upper limit of normal satisfying the diagnostic criteria for pancreatitis. The patient had no known risk factors for pancreatitis and with the rapid decrease in lipase levels after discontinuation of treatment, the likelihood of pazopanib-induced pancreatitis becomes more evident. This has been shown in similar cases where discontinuation of treatment led to rapid decreases in lipase levels [4]. The mechanism of pazopanib-induced pancreatitis is largely unknown; however, several theories have been hypothesized. One possible mechanism is that TKIs like pazopanib may induce microvascular is- 
chemia due to their anti-angiogenic effect, placing the patient at risk for pancreatic inflammation. Another theory suggests that TKI-induced decreased gastrointestinal motility may cause reflux of duodenal contents, and activation of pancreatic enzymes. Because TKIs inhibit vascular endothelial growth factor (VEGF), it is also possible that the resulting loss of acinar cells induces an increase in pancreatic enzymes. While all theories are only speculation, it is known that pazopanib relation to an increase in pancreatic enzymes is not dose-dependent. Further researches on the specific mechanisms are needed to comprehend the pathogenesis of TKI-induced pancreatitis [4].

In the event pancreatitis develops following TKI administration for renal cell carcinoma, TKI treatment should be discontinued promptly. Even upon recovery, TKIs are not suggested for reinstatement, and an alternate treatment for renal cell carcinoma treatment should be considered [5].

Pazopanib has been shown to be an effective treatment option for renal cell carcinoma and oncologists and other medical professionals should recognize acute pancreatitis as a possible side effect of pazopanib therapy. Due to this rare but severe side effect, patients should be educated on symptoms and semiregular monitoring of pancreatic enzymes may be beneficial.

\section{Financial Disclosures}

We do not have any financial disclosures nor have we received any funding.

\section{Conflicts of Interest}

There are no conflicts of interest.

\section{References}

1. Cappell MS. Acute pancreatitis: etiology, clinical presentation, diagnosis, and therapy. Med Clin North Am. 2008;92(4):889-923, ix-x.

2. Tenner S, Baillie J, DeWitt J, Vege SS. American College of Gastroenterology guideline: management of acute pancreatitis. Am J Gastroenterol. 2013;108(9):1400-1415; 1416.

3. Kawakubo K, Hata H, Kawakami H, Kuwatani M, Kawahata S, Kubo K, Imafuku K, et al. Pazopanib-Induced Severe Acute Pancreatitis. Case Rep Oncol. 2015;8(2):356358.

4. Russano M, Vincenzi B, Venditti O, D'Onofrio L, Ratta R, Guida FM, Tonini G, et al. Pazopanib and pancreatic toxicity: a case report. BMC Res Notes. 2015;8:196.

5. Schmidinger M, Larkin J, Ravaud A. Experience with sunitinib in the treatment of metastatic renal cell carcinoma. Ther Adv Urol. 2012;4(5):253-265. 